\title{
Varianten in der Produktionstechnologie der Stropharia sp. in Ungarn
}

\author{
SÁNDOR BALÁZS und ANDRAS KOVÁCS
}

\begin{abstract}
BALÁZS, S. \& KOVÁCS, A. 1978: Varianten in der Produktionstechnologie der Stropharia Sp. in Ungarn. - Karstenia 18 (suppl.).

Im Laufe der Versuche wurde die Zusammensetzung des Substrates (das vorbehandelte und unmanipulierte Stroh verschiedener Getreidearten) untersucht, ausserdem mussten wir auch die beim Champignon gebrauchte Zudeckung ändern, nachdem die Reaktion dieser fur den Strophariapilz nicht geeignet war.

Unter den Kl imabedingungen Ungarns hat der Anbau des Pilzes zwischen den Monaten Mai und Juni wegen der grossen $\mathrm{Hitze}$ und des damit verbundenen Wasserverlustes ausserordentlich viel Schwierigkeiten bereitet und Arbeitsmehrbedarf gefordert. Deswegen haben wir mit neuen Aussatsterminen Versuche durchgefuhrt.

Das ganze Jahr hindurch wurde zweiwöchentl ich eine Neusaat vorgenommen, infolgedessen wurden bel der spaten Herbstsaat zu Ende September - Oktober günstige Ergebnisse bestatigt. Die zu dieser Zeit angebaute Stropharia durchwebt bei sehr niedriger Temperatur frei von anderen kurrenten Organen das Substrat. Bei solcher winterlichen Durchwebung wurde sozusagen auch die Pasteurisierung des Substrates gelost. Mit dieser Aussaatmethode wurde die Kultur wesentlich einfacher und die Durchschnittsertrăge stiegen.

Gegenwărtig kann die Durchwebefahigkeit bei niedriger Temperatur zum zeitgemassen Anbau angewandt werden. Der wăhrend dem Winter durchwebte Năhrboden kann in Kisten oder in polyethylen Sacken am Ende des Winters, im Laufe des Frühjahres in geheizten Raumen solyethylen Sacken am Ende des Winters,

Die Überwinterungsmethode kann in erster Linie in Kleinbetrieben wigtschaftlich angewandt werden, da man auch ohne grössere Investierung mit 8-12 kg pro m Ertrag rechnen kann.
\end{abstract}

S. Baläzs \& A. Koväcs, Forschungsinstitut für Gemüsebau, Kecskemēt, Ungarn.

Der Pilz Stropharia rugoso-annulata wurde in Ungarn erst vor einigen Jahren in Kultur genommen. Während in modern ausgerusteten Betrieben jährlich etwa 3000 t Champignons, Agaricus bisporus geerntet werden, haben andere Pilzarten in der Produktion fast keine Rolle gespielt. Um 1950 wurden in Ungarn die ersten Anbauversuche mit anderen Pilzarten begonnen. Neue, schmackhafte Gerichte für die Kulche und billiger herstellbare Pilze wurden gesucht. Da die Agaricus-Arten hauptsächlich auf Pferdemist oder auf synthetischem Kompost gedeihen, dessen Anschaffung ziemlich kostspielig ist, wurden Arten gesucht, die auch auf in grossen Mengen vorkommenden billigen landwirtschaftlichen Nebenprodukten oder sonstigem Abfall mit Erfolg angebaut werden können. So wurden Pleurotus ostreatus und $P$. florida in Kultur genommen. Die Produktion von pleurotus nimmt ständig zu. Man hat sich allmählich an den Geschmack gewöhnt; der Aussenhandel beginnt sich auch für die Sache zu interessieren, so dass die Pleurotus-Arten langsam mit den AgariousArten zu konkurrieren anfangen.

Versuche wurden und werden in Ungarn auch mit einer sehr wärmeliebenden Art, Volvarielza volvacea, durchgefürt. Der Anbau auf angereichertem Stroh nimmt vom Anlegen bis zur Ernte - nur 30 Tage in Anspruch. Wegen des hohen Wärmebedarfs könnte der Anbau in der Sommerzeit in mit Thermalwasser versorgten Gewächs- häusern vorgenommen werden. Infolge seines ausgezeichneten Geschmacks, seiner Anspruchslosigkeit dem Substrat gegenuber und kurzer Anbauzeit sollten die in Asien ublichen, völlig auf Handarbeit ruhenden Anbaumethoden modernisiert werden um auch in unseren Grossbetrieben Anwendung finden zu können. Unserer Meinung nach könnte der Pilz die wertvollste angebaute Spezies sein, wenn wir genügende Kenntnisse über den Anbau sammeln.

Seit einigen Jahren beschäftigen wir uns intensiver mit dem Anbau von s. rugoso-annulata. Es handelt sich um eine leicht und billig herstellbare Art, die bisher nirgends auf grösseren Flächen angebaut worden ist.

In Ungarn hat der Anbau den in der DDR ausgearbeiteten Methoden gefolgt.

Als Substrat wird $10 \mathrm{~kg}$ lufttrockenes Stroh/ $\mathrm{m}^{2}$ genommen. Vor dem Anlegen wird das Stroh befeuchtet, so dass es etwa 70 \% Feuchtigkeit enthält, dann 20-30 cm dick ausgebreitet und mit 1 Liter $/ \mathrm{m}^{2}$ Brut beimpft. Das Anlegen beginnt Ende Mai, Anfang Juni. Das Myzel braucht lange Zeit - nach bisherigen Erfahrungen etwa 4 Wochen - bis es das Substrat ganz durchzieht. Erst danach kann die Deckerde 4-5 cm dick ăhnlich wie bei Agaricus, aufgebracht werden. Vor dem Aufbringen der Deckerde kann das Substrat mit leichtem Material, das eine gute Durchlüftung 
ermöglicht und zugleich vor Austrocknung schützt, abgedeckt werden. Nach dem Abdecken erfolgt eine relativ lange Anwachsperiode ( $8-9$ Wochen), bis die ersten Fruchtkörper, wahrscheinlich vom Wetter beeinflusst, Ende August erscheinen.

In Ungarn ist das Wetter zwischen Mai und oktober im allgemeinen sehr warm; Stropharia erträgt die hohen Temperaturen ziemlich gut, die Pflegearbeiten sind jedoch in der Hitze wesentlich schwieriger durchzufuhren als unter geregelten Klimaverhältnissen. Die starken Temperaturschwankungen wirken sich ungünstig auf die Feuchtigkeit der Deckerde, des Substrats und der Luft aus. Unregelmässige Wasserversorgung und schwankende Luftfeuchtigkeit verzögern das Wachstum des Myzels. Wir nehmen an, dass diese Umstănde wohl dabei mitspielen, dass die Vegetationsperiode von Stropharia in extensivem Anbau mit den in der DDR ausgearbeiteten Methoden viel länger dauert, als die der erwahnten Agaricus, Pleurotus oder Volvarielza-Arten. Auf diese Weise schwankt der Ertrag erheblich: $1-2 \mathrm{~kg} / \mathrm{m}^{2}$ aber auch $8-10 \mathrm{~kg} / \mathrm{m}^{2}$ (10 kg lufttrockenes stroh).

In unseren, seit 6 Jahren fortgesetzten Versuchen um den Ertrag zu sichern und zu erhöhen werden auf forgende Fragen Antwort gesucht:

I. Welche Forderungen stellt die Stropharia-Art der Umwelt gegenüber (Wärme, Wasser, Substrat, Deckerde)?

II.Welche Anbauperiode scheint im extensiven Anbau in Ungarn am günstigsten zu sein? Können Veränderungen in den Anbaumethoden zu sicheren, hohen Erträgen fuhren?

Folgonde Teilergebnisse wurden ermittelt:

I. Um den Warmebedarf von Stropharia bestimmen zu können, wurde das Myzelwachstum in verschiedenen Temperaturen untersucht. Ausser dem Optimum wurden auch die Maximum- und Minimum-Werte gesucht, wo das Myzel noch $\mathrm{zu}$ wachsen imstande ist. Die Ergebnisse sind in $\mathrm{Abb}$. 1. zusammengefasst.

Versuchtsbeobachtungen zeigen, dass das Myzel um $25^{\circ} \mathrm{C}$ sehr intensiv wächst, unter $-5^{\circ} \mathrm{C}$ oder uber $+35^{\circ} \mathrm{C}$ aber abstirbt (Abb. 1). Es wurde erwiesen, dass im Gegenteil zu Agaricus, keine Anomalien (Myzel-Absterben, Degeneration) bei niedrigeren Temperaturen $\left(5-10^{\circ} \mathrm{C}\right)$ vorkommen. Bei höheren Temperaturen sind aber Unkrautpilze erschienen, deren Vegetationszeit viel kürzer ist, als die der stropharia und so ihren Lebenszyklus von der Fruchtkörperbildung der Stropharia beenden (Coprinus sp., Peziza sp., Volvarielza sp.).

Versuche haben erwiesen, dass Abweichungen vom Temperaturoptimum die Vegetationszeit mehr oder weniger verlängern.

Nach Beobachtungen soll das Myzel auf Substrat mit $70 \%$ Wassergehalt am besten gedeihen, bei einem Wassergehalt von unter $40 \%$ oder uber $80 \%$ hört das Wachstum auf (Abb. 2).

In Substrat-Versuchen wurden verschiedene Getreidestroharten (Weizen, Gerste, Triticale, Roggen),
Abb. 1. Der Einfluss verschiedener Temperaturen auf das Längewachstum des Myzels ( $\mathrm{mm}$ ) (Beobachtungszeit: 12 Tage). Budapest, 1973.

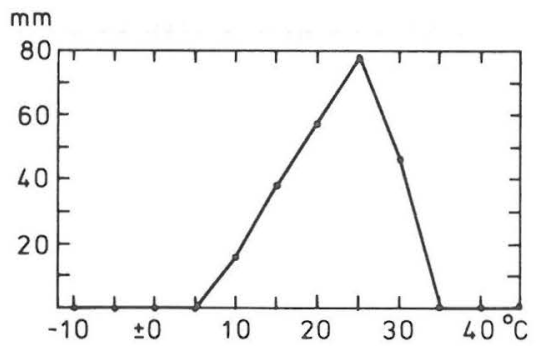

Abb. 2. Myzel-Wachstum auf Substrat mit unterschiedlichem Wassergehalt (in 21 Tagen).

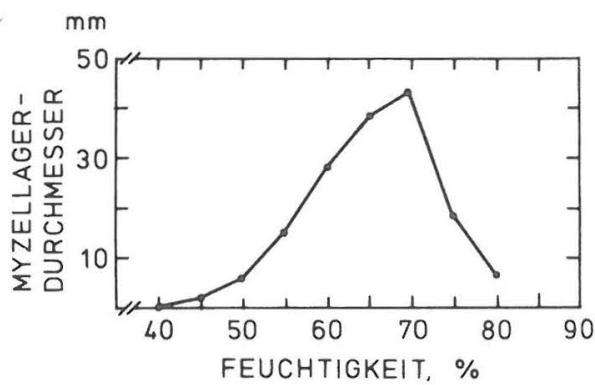

Strohkompost, frisches Stroh, aber auch Pferdemistkompost und abgetragener Champignonkompost miteinander verglichen.

Die Ergebnisse haben die Beobachtungen von Puschel bestatigt, dass stropharia ausschliesslich auf Stroh gedeint und sich die Beimischung von Mineraldunger zur Anreicherung des Substrats nachteilig auswirkt. Bei den verschiedenen Strohvarianten ist kein Unterschied im Ertrag zu finden. Ein- oder zwei-jăhiriges Stroh bewahrt sich gleich gut, wenn es nur gesund ist. Die Stropharia-Arten nutzen das Stroh gut aus.

Im mehr-jăhrigen Durchschnitt haben wir im Herbstanbau

0.8-1 kg Frischware bei der Sorte Gelb,

$0.6-0.8 \mathrm{~kg}$ Frischware bei der Sorte Winnetou,

0.3-0.6 kg Frischware bei der Sorte Gartenriese auf

$1 \mathrm{~kg}$ lufttrockenem stroh geerntet.

In den Versuchen wurde das Stroh

1. I Stunde lang in warmes Wasser getaucht $\left(60^{\circ} \mathrm{C}\right)$ und die Behandlung am folgenden Tag wiederholt

2. eine halbe stunde lang mit Dampf $\left(90^{\circ} \mathrm{C}\right)$ behandelt dann abgekuhlt und die Behandlung am folgenden Tag wiederholt.

Bei der Kontrolle wurde das Stroh nur mit kaltem Wasser befeuchtet. Die Zeit bis zum Erscheinen 
der Fruchtkörper hat sich verkürzt; auf dem eingeweichten Substrat erschienen die ersten Fruchtkörper von der Sorte abhängig - nach 58-70 Tagen, auf dem Eedämpften Substrat nach 70-85 Tagen, auf dem der Kontrolle nach 85-100 Tagen. Im Ertrag sind keine wesentliche Unterschiede zu finden; die Reihenfolge ist jedoch die gleiche geblieben: auf dem eingeweichten Substrat wurden mehr Pilze geerntet, als auf dem gedämpften oder auf dem mit kaltem wasser behandelten.

In den Deckerde-Versuchen wurden die sich im Agaricus-Anbau gut bewährten Torfhumus-Deckerden mit anderen Deckerden vom $\mathrm{pH} 7$ verglichen (Abb. 3).

Um den gewünschten pH-Wert bekommen zu können, wurden sägemehl und saurer Torf in verschiedenen Konzentrationen zur humusreichen Gartenerde gemischt.

Es wurde eindeutig erwiesen, dass die bei uns gebräuchliche Gartenerde ( $\mathrm{pH} 7.6)$ im Verhaltnis 80:20 mit sägemehl vermischt werden muss, um eine günstige Reaktion in der Deckerde herbeizufuhren. Auf einer solchen Deckerde erscheinen die Fruchtkörper gleichmässig auf der ganzen Fläche des Beetes. Die mit Torf vermischte Deckerde besitzt die gleichen Eigenschaften, wie die Frühbeet- und Săgemehl-Mischung, kann aber kaum Anwendung finden, da Torf in Ungarn nur an wenigen Orten vorkommt. Nach unseren Beobachtungen sollte der pH-Wert in der Deckerde und im Substrat gleichsam sauer sein. Der Pilz stellt aber den $\mathrm{pH}$-Wert des Substrats selbts ein.

Abb. 3. Der Einfluss von pH-Wert und Feuchtigkeit der Deckerde auf das Erscheinen von Fruchtkörpern. Kecskemét, 1974.

\begin{tabular}{|c|c|c|c|c|}
\hline Benennung & $\%$ & $\mathrm{pH}$ & $\begin{array}{l}\text { Feuchtig- } \\
\text { keit } \%\end{array}$ & $\begin{array}{l}\text { Erscheinen der } \\
\text { Fruchtkörper }\end{array}$ \\
\hline Frühbeeterde & 100 & 7.6 & 31.0 & am Rande des Beetes \\
\hline Frühbeeterde + & 34 & & & \\
\hline Torf (alkalisch) + & 33 & 8.0 & 30.0 & am Rande des Beetes \\
\hline Kalk & 33 & & & \\
\hline Frulhbeeterde + & 70 & & & \\
\hline Sägemehl + & 20 & 7.2 & 31.4 & auf der ganzen Ober- \\
\hline Torf (sauer) & 10 & & & fläche des Beetes \\
\hline Fruthbeeterde + & 80 & & & \\
\hline Sägemehl & 20 & 7.3 & 28.3 & $\begin{array}{l}\text { auf der ganzen Ober- } \\
\text { fläche des Beetes }\end{array}$ \\
\hline
\end{tabular}

II. Um den günstigsten Anlegezeitpunkt ermitteln zu können, wurden Versuche in monatlichen Etappen, im Spätherbst, Winter und Fruhling, in Grube, Gewächshaus oder unter Folie durchgefürt. Herbstanbau (in Grube) und Frülingsanbau wurden miteinander verglichen.
Die Vegetation nimmt beim Anlegen Mitte Mai 160 Tage in Anspruch; bei der Septemberkultur 300 Tage und bei der Novemberkultur 240 Tage. Im Fruhling dauert die Vegetation fast halb so lange, wie im Herbst, erfordert aber wesentlich mehr Pflege. Der Einfluss des Anbauzeitpunktes auf den Ertrag geht aus Abb. 4 hervor.

Es zeigt sich, dass die Ertragsmenge beim Anbau am 1. September dreimal und die im Oktober viermal den Ertrag des Frühlingsanbaues ubertrifft.

Im Spätherbst könnten wir unter extensiven Bedingungen - zum Beispiel in ungeheizten Folienhäusern in Foliensäcken - (dem Agarious-Anbau gleich) das Substrat vom Myzel durchwachsen lassen. Ende Januar kann dann die Kultur in geheizten Räumen $\left(20-25^{\circ} \mathrm{C}\right)$ oder im Gewächshaus fortgesetzt werden. Es ist denkbar, dass auf diese Weise stropharia auch

Abb. 4. Der Einfluss des Anlegezeitpunktes auf den Ertrag der Sorte Gelb $\left(\mathrm{kg} / \mathrm{m}^{2} / 10 \mathrm{~kg}\right.$ trockenes Stroh) in 1974-1976.

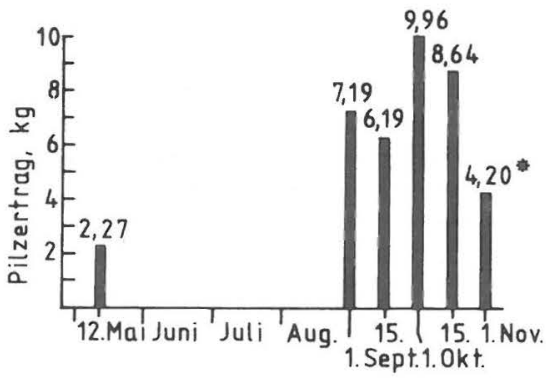

* Daten aus einem Jahr

zu Grossbetrieben Einlass finden kann. Diesbezügliche Versuche müssen noch weitergefuhrt werden. Bisherige Versuche haben erwiesen, dass 's. rugoso-annulata in Kleinbetrieben billig herzustellen ist. Der Herbstanbau bietet auch in Grossbetrieben Möglichkeiten fur einen wirtschaftlichen Anbau. Die Anbaukosten sind im Vergleich zu den anderen, kultivierten Pilzarten die niedrigsten. Wenn die Fragen der Grossproduktion gelöst werden können, wird die Auswahl der essbaren Pilze mit einer neuen Art erweitert. 